\title{
Level repulsion in nanophotoluminescence spectra from single GaAs quantum wells
}

\author{
G. von Freymann,* U. Neuberth, M. Deubel, and M. Wegener \\ Institut für Angewandte Physik, Universität Karlsruhe (TH), Wolfgang-Gaede-Straße 1, 76131 Karlsruhe, Germany \\ G. Khitrova and H. M. Gibbs \\ Optical Sciences Center, University of Arizona, Tucson, Arizona 85721
}

(Received 12 December 2001; published 22 May 2002)

\begin{abstract}
In a recent paper, Intonti et al. attributed [Phys. Rev. Lett. 87, 076801 (2001)] a 3-meV peak in the autocorrelation spectrum of low-temperature nanophotoluminescence spectra of a single disordered GaAs quantum well to level repulsion, i.e., to the statistical analog of an avoided crossing due to overlapping wave functions in the disorder potential. Our data, which reproduce their findings very nearly, are taken to an additional test employing filter functions, which clearly shows that the $3-\mathrm{meV}$ peak is associated with lowenergy states - in striking contrast to the level repulsion scenario. By a careful analysis of the high-energy states, however, we are able to identify a second peak around $1.5 \mathrm{meV}$ which we attribute to level repulsion. The experiments are compared with simple model calculations, which support our interpretation.
\end{abstract}

DOI: 10.1103/PhysRevB.65.205327

PACS number(s): 78.55.Cr, 07.79.Fc, 73.20.Fz, 78.67.De

\section{INTRODUCTION}

A striking phenomenon of disordered systems is the fact that the energy levels of a quantum-mechanical particle in a random potential are not simply random. More precisely, a white-noise real-space potential $V(\vec{r})$, which has a correlation function $\left\langle V(\vec{r}) V\left(\vec{r}^{\prime}\right)\right\rangle=\delta\left(\vec{r}-\vec{r}^{\prime}\right)$, leads to energy levels which are not just white noise. Random matrix theory predicts energy-level repulsion, meaning that it is less likely to find adjacent pairs of individual energy levels which are energetically nearby in energy than it is to find pairs which are further apart in energy. The atomic scale roughness of semiconductor quantum wells (QW's) leads to a disorder potential for excitons in the QW $x y$-plane, and represents a nice model system to study these effects. In a recent letter, ${ }^{1}$ Intonti et al. observed a maximum around an energy difference of $\Delta E=3 \mathrm{meV}$ in the average autocorrelation functions ${ }^{2}$ of nano-photoluminescence (PL) spectra of a single 3-nm-thin GaAs QW. They attributed the $3-\mathrm{meV}$ peak to an interplay between level repulsion-which leads to a dip at small energies - and correlations in the disorder potential. Together these make an energy separation of the PL lines of $3 \mathrm{meV}$ more likely than others. Intonti et al. found good agreement with the theoretically expected statistics of the eigenenergies in a disorder potential which is a convolution of white noise with a Gaussian.

We reproduce the experimental findings of Intonti et al. on several samples under very nearly identical conditions. However, by applying filter functions to the data we find that the 3-meV peak is not consistent with level repulsion. Also, again by virtue of using filter functions, we are able to identify an additional peak around $\Delta E=1.5 \mathrm{meV}$ corresponding to high-energy states in the PL spectrum which we attribute to level repulsion. Moreover, by introducing the technique of spatial autocorrelations of the measured PL maps, we are able to demonstrate that these high-energy states are actually more extended than the low-energy ones.

Level repulsion is the statistical analog of an avoided crossing: otherwise accidentally degenerate energy levels tend to split because of the finite overlap of the wave functions. This trend is obviously pronounced for the more extended high-energy levels and weaker for the more localized low-energy states of the spectrum. How can we test this important characteristic energy dependence? As we have pointed out, ${ }^{2}$ we can multiply the measured individual PL spectra, $I_{n}(\hbar \omega)$ by filter functions of the form

$$
f(\hbar \omega) \propto \exp \left( \pm \hbar \omega / E_{0}\right),
$$

with a filter parameter $E_{0}$ and a photon energy $\hbar \omega$, i.e., $I_{n}(\hbar \omega) \rightarrow f(\hbar \omega) \times I_{n}(\hbar \omega)$. For the $+(-)$ sign, the high(low-) energy states are emphasized. Thus, for $+(-)$ we expect the $3-\mathrm{meV}$ peak to remain or increase (decrease), which is what we indeed find in one-dimensional numerical calculations strictly following the lines of the theoretical calculations of Ref. 1 for level repulsion (see Sec. III).

This paper is organized as follows: Section II presents our experimental results obtained from four different samples with some qualitative discussion. In Sec. III we compare these experimental findings with simple numerical calculations, and show that it is crucial not only to account for atomic scale roughness of the QW but also for the monolayer islands-even if the monolayer islands are not apparent from the average PL spectra. Furthermore, it will become obvious that a rich variety of behaviors results from rather small variations of the parameters of the disorder potential. In other words, we are able to draw one consistent picture for all the samples investigated here and in previous work.

\section{EXPERIMENT}

In the following, we show data sets based on $N$ individual nano-PL spectra. ${ }^{2,1}$ Depending on the requirements, $N$ varies between 6400 and 12800. The design of all the samples discussed in this paper is summarized in Table I. The first sample to be discussed, cat81 (Fig. 1), is very nearly identical to the sample shown in Ref. 1. It is a single, 3.5-nm-thin, growth-interrupted GaAs QW with superlattice barriers 
TABLE I. Growth parameters of the four samples investigated.

\begin{tabular}{|c|c|c|}
\hline & cat $81 /$ cat54 & cat $84 /$ cat 83 \\
\hline cap layer & 10-nm GaAs & $3.5 \mathrm{~nm} \mathrm{GaAs}$ \\
\hline barrier & $\begin{array}{c}\text { 37-nm } \mathrm{Al}_{0.33} \mathrm{Ga}_{0.66} \mathrm{As} \\
\text { grown as a } \mathrm{SL}\end{array}$ & $\begin{array}{c}75 \mathrm{~nm} \mathrm{Al}_{0.33} \mathrm{Ga}_{0.66} \mathrm{As} \\
\text { grown as a } \mathrm{SL}\end{array}$ \\
\hline GI & $180 \mathrm{~s}$ after $\mathrm{GaAs}$ & $130 \mathrm{~s} / 240$ s after $\mathrm{GaAs}$ \\
\hline QW & $3.5 \mathrm{~nm} \mathrm{GaAs}$ & $5.0 \mathrm{~nm} \mathrm{GaAs}$ \\
\hline barrier & $\begin{array}{c}134 \mathrm{~nm} \mathrm{Al}_{0.33} \mathrm{Ga}_{0.66} \mathrm{As} \\
\text { grown as } \mathrm{SL}\end{array}$ & $\begin{array}{c}75 \mathrm{~nm} \mathrm{Al}{ }_{0.33} \mathrm{Ga}_{0.66} \mathrm{As} \\
\text { grown as } \mathrm{SL}\end{array}$ \\
\hline cap & none & $3.5 \mathrm{~nm}$ \\
\hline etch stop & $150 \mathrm{~nm} \mathrm{Al}{ }_{0.85} \mathrm{Ga}_{0.15} \mathrm{As} /$ none & $150 \mathrm{~nm} \mathrm{Al} \mathrm{Al}_{0.85} \mathrm{Ga}_{0.15} \mathrm{As}$ \\
\hline buffer & $750 \mathrm{~nm} \mathrm{GaAs}$ & $450 \mathrm{~nm} \mathrm{GaAs}$ \\
\hline substrate & $0^{\circ} \mathrm{GaAs}(100)$ & $0.6^{\circ} / 0^{\circ} \mathrm{GaAs}(100)$ \\
\hline
\end{tabular}

[4 ML of AlAs, no interruption, 8 ML of GaAs, 180-s growth interruption (GI), etc.], grown by molecular-beam epitaxy on a (100) GaAs substrate. The GaAs film is $47 \mathrm{~nm}$ away from the sample surface. The excitation power (at $\hbar \omega_{\text {exc. }}$. $=1.96 \mathrm{eV}$, from a He-Ne laser as in Ref. 1 or at $\hbar \omega_{\text {exc. }}$. $=1.73 \mathrm{eV}$, from a Ti:sapphire-laser as indicated) sent into the optical monomode fiber with a nanometer tip at its end ${ }^{2}$ is $P=100 \mathrm{nW}$ (a factor of 8 lower than in Ref. 1), and the sample temperature is $T=10 \mathrm{~K}$, unless stated otherwise. The light is collected by the same uncoated fiber tip and is sent into a $0.5-\mathrm{m}$ grating spectrometer. In this fashion, the spatial resolution is $300 \mathrm{~nm}$, and the spectral resolution $150 \mu \mathrm{eV}$. As also explicitly shown in Ref. 1, we find no dependence of our results on temperature and excitation power between 5 and $30 \mathrm{~K}$, and from $25 \mathrm{nW}$ to $1.6 \mu \mathrm{W}$, respectively (to be shown in Fig. 7). The latter is also expected by a simple estimate of the average number of excitons, $N_{x}$, under the $(300 \mathrm{~nm})^{2}$ spot as $N_{x}=P[1-\exp$ $\left.\left(-\alpha L_{z}\right)\right] \tau /(\hbar \omega)$, with an estimated absorption coefficient $\alpha=2 \times 10^{4} \mathrm{~cm}^{-1}$, and a QW thickness $L_{z}=3.5 \mathrm{~nm}$ (for excitation below the barrier absorption, Ti:sapphire); $P$ $=100 \mathrm{nW}$, the independently measured photoluminescence

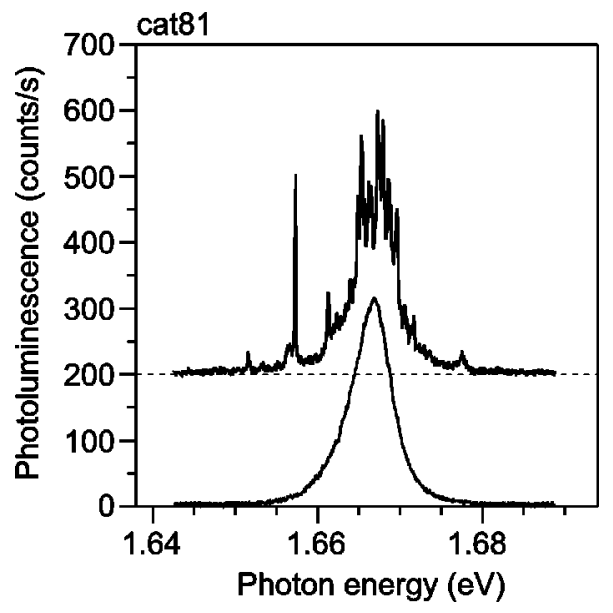

FIG. 1. Individual (top) and average (bottom) photoluminescence spectrum of sample cat $81 ; N=6400, T=10 \mathrm{~K}$, and $\hbar \omega_{\text {exc. }}$. $=1.96 \mathrm{eV}$.
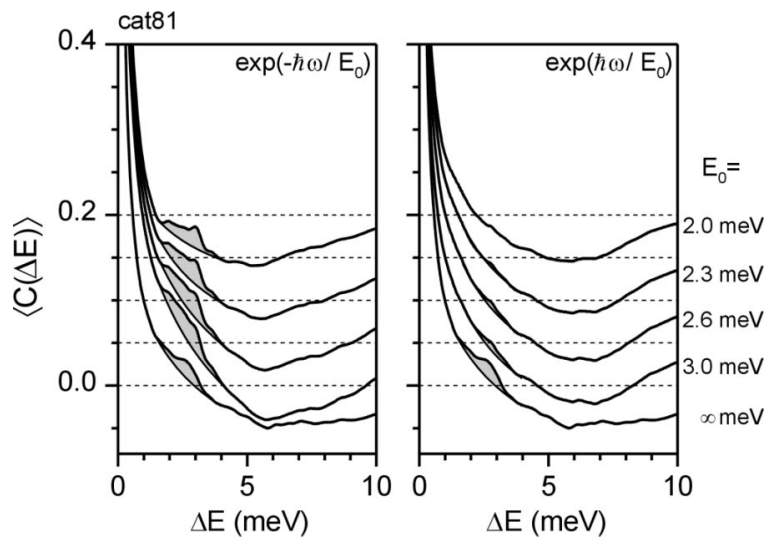

FIG. 2. Average autocorrelation spectra of sample cat81; $N$ $=6400, T=10 \mathrm{~K}$, and and $\hbar \omega_{\text {exc. }}=1.96 \mathrm{eV}$, for different filter functions $f(\hbar \omega)=\exp \left( \pm \hbar \omega / E_{0}\right)$ which are multiplied with the individual PL spectra. The filter parameter $E_{0}$ is as indicated, and $E_{0}=\infty \mathrm{meV}$ is equivalent to no filter. Spectra are vertically displaced for clarity. Also, the relevant feature is highlighted by the gray areas to guide the eye. Note that the feature around $\Delta E$ $=3 \mathrm{meV}$ disappears for decreasing $E_{0}$ on the right-hand sides while it remains nearly constant on the left-hand sides. The same results are obtained for $\hbar \omega_{\text {exc. }}=1.73 \mathrm{eV}$ (not shown).

decay time under these conditions is $\tau=175 \mathrm{ps}$, and the excitation photon energy is $\hbar \omega_{\text {exc. }}=1.73 \mathrm{eV}$. This leads to $N_{x}=0.44$. As this average number of less than one exciton within an area of $(300 \mathrm{~nm})^{2}$ is distributed over several tens of individual potential minima, we can safely exclude manyexciton effects under these conditions. Carrier diffusion (which we have neglected in the estimate) will further reduce this number.

The autocorrelation analysis consists of five steps and is similar to Refs. 2 and 1: (1) the individual spectra $I_{n}(\hbar \omega)$ are normalized to equal spectral integral; (2) the average spectrum is subtracted from each individual spectrum (this subtracts uncorrelated backgrounds), and $\delta I_{n}(\hbar \omega)=I_{n}(\hbar \omega)$ $-\left\langle I_{n}(\hbar \omega)\right\rangle$; (3) the individual autocorrelation spectra are computed from this $C(\Delta E)=\int \delta I_{n}\left(\hbar \omega^{\prime}\right) \delta I_{n}\left(\hbar \omega^{\prime}\right.$ $+\Delta E) d \hbar \omega^{\prime}$ and (4) are averaged over a complete data set. (5) For convenience, the averaged autocorrelation function $\langle C(\Delta E)\rangle$ is normalized to unity at $\Delta E=0$.

Figure 2 shows the measured behavior on sample cat81. It has a 5.6-meV average PL linewidth (Fig. 1). For the minus sign of the filters [the left-hand side (LHS) of Fig. 2], the 3 -meV peak becomes even more pronounced, while it rapidly disappears for the plus sign [right-hand side (RHS) of Fig. 2]. This clearly demonstrates that the $3 \mathrm{meV}$ autocorrelation peak arises predominantly from low-energy states rather than from high-energy states-in striking contrast to the level repulsion scenario. This trend is also observed on another growth-interrupted GaAs QW sample with a 5-nm well width, cat84, grown with superlattice barriers (4 ML of AlAs, no interruption, $8 \mathrm{ML}$ of GaAs, 130-s growth interruption, etc.). The QW is $78.5 \mathrm{~nm}$ underneath the surface. This sample also does not show immediate evidence of monolayer island splitting, ${ }^{3,4}$ and has an average PL linewidth of 12 meV (Fig. 3), which is very nearly similar to the sample of 


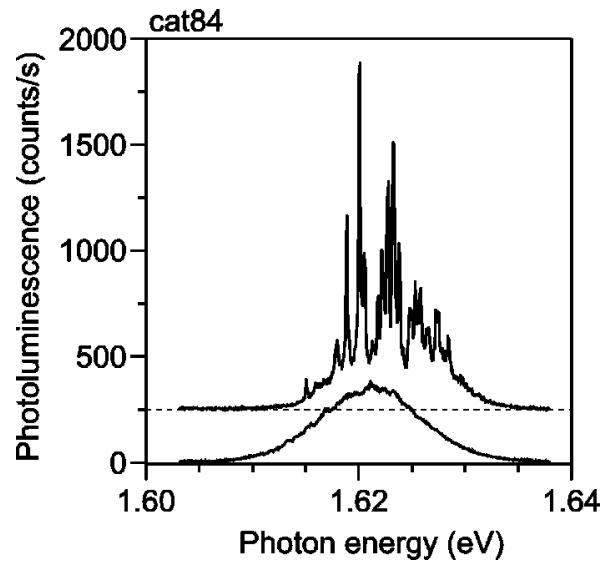

FIG. 3. Individual (top) and average (bottom) photoluminescence spectra of sample cat84; $N=6400, T=10 \mathrm{~K}$, and $\hbar \omega_{\text {exc. }}$. $=1.96 \mathrm{eV}$.

Ref. 1. The data on samples cat84 (Fig. 4) and cat81 (Fig. 2) indicate that the disorder potential is more complex than just white noise with a certain correlation length.

This becomes even more obvious on two samples, cat54 and cat83, we have studied which do show monolayer splitting in the average PL spectra. Sample cat54 (Fig. 5) is the same as sample cat 81 except for the etch-stop layer. Because of the clear distinction between the different monolayers in the average PL spectrum of sample cat54 (Fig. 5), it is advantageous to employ rectangular spectral filters rather than the exponential filters used for sample cat84 in Fig. 4. We define two filters, labeled $n+1$ ML and $n$ ML in Fig. 5, corresponding to the different ML regions. For the lowenergy part of the PL spectrum we again find an autocorrelation peak around $\Delta E=3 \mathrm{meV}(n+1 \mathrm{ML}$ in Fig. 6), consistent with our above findings on samples cat 81 and cat 84 . For the high-energy region ( $n$ ML in Fig. 6), where we expect to find level repulsion, only a broad shoulder is observed as for sample cat 84 . This is due to the fact that the width of the individual PL lines in this sample smeares out the expected level repulsion dip. This will be different in sample cat 83 .


FIG. 4. Average autocorrelation spectra of sample cat84, $N$ $=6400, T=10 \mathrm{~K}$, and $\hbar \omega_{\text {exc. }}=1.96 \mathrm{eV}$, for different filter functions as in Fig. 2. The same results are obtained for $\hbar \omega_{\text {exc. }}$ $=1.73 \mathrm{eV}$ (not shown).

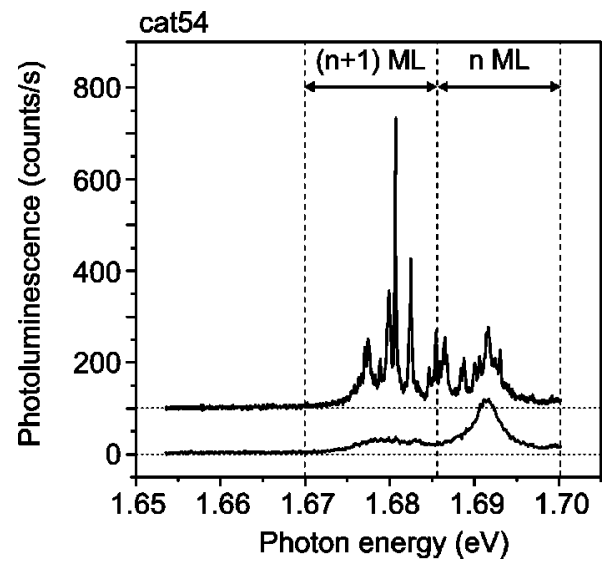

FIG. 5. Individual (top) and average (bottom) photoluminescence spectra of sample cat54, $N=6400, T=10 \mathrm{~K}$, and $\hbar \omega_{\text {exc. }}$. $=1.73 \mathrm{eV}$.

Before we come to this interesting point, for the example of sample cat54 we demonstrate that not only the individual spectra (as already shown in Ref. 1) but also the autocorrelation functions show no significant dependence on the incident excitation power (Fig. 7). A reduction of the incident power down to $25 \mathrm{nW}$ does not change the correlation maximum of the $(n+1)$ ML peak. ${ }^{8}$ For very large excitation, this feature gradually disappears. The same holds for increasing sample temperature (RHS of Fig. 7). This intensity independence rules out biexciton effects as a possible origin of the autocorrelation maxima for all the samples investigated here.

Sample cat83 (Fig. 8) differs from sample cat84 only in two respects, both favorable to the formation of large islands: the growth interruption after the QW was $240 \mathrm{~s}$, and the substrate was untilted compared with the $0.6^{\circ}$ tilt for sample cat 84 . Because of the highly structured average PL spectrum of sample cat83 (Fig. 8), we employ rectangular spectral filters as introduced for sample cat54. We define three filters, labeled low, middle (mid), and high in Fig. 8. For the lowenergy part of the PL spectrum we again find the autocorrelation peak (low in Fig. 9), consistent with our above findings on samples cat84, cat 81 , and cat54, but slightly shifted toward lower $\Delta E$ than $3 \mathrm{meV}$. In contrast to this, we find a different line shape as well as a different position of the peak



FIG. 6. Average autocorrelation spectra of sample cat54, N $=6400, T=10 \mathrm{~K}$, and $\hbar \omega_{\text {exc. }}=1.73 \mathrm{eV}$, for rectangular filter functions as in Fig. 5. 


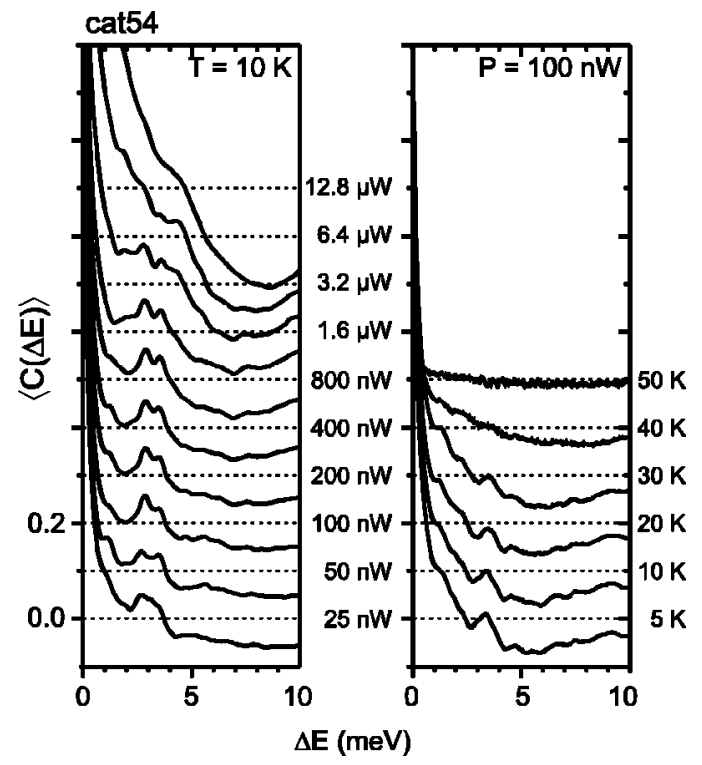

FIG. 7. Average autocorrelation spectra of sample cat54, $N$ $=400$ and $\hbar \omega_{\text {exc. }}=1.73 \mathrm{eV}$, for the $(n+1) \mathrm{ML}$ area. Temperature and excitation power are as indicated.

$(1.5 \mathrm{meV})$ in the autocorrelations for the high filter. The mid filter does not show any interesting autocorrelation features.

As pointed out above, it is expected that level repulsion corresponds to spatially more extended states. To test this crucial aspect, we show PL maps of the two different spectral regions low and high in Fig. 10(a). It is obvious that the low maps exhibit resolution-limited small spots, while the images corresponding to high maps exhibit more extended bright

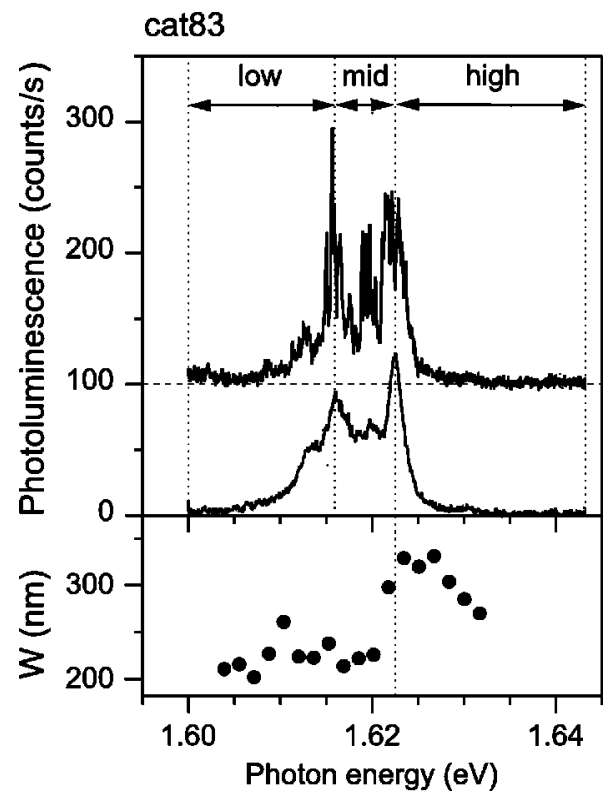

FIG. 8. Individual (top) and average (middle) photoluminescence spectrum of sample cat83, $N=12800, T=10 \mathrm{~K}$, and $\hbar \omega_{\text {exc. }}=1.73 \mathrm{eV}$. The circles (bottom) denote the half-width at half maximum $W$ of the spatial autocorrelations obtained from PL maps similar to those in Fig. 10. Note that they tend to be more extended in real space for the high region than for the low region.


FIG. 9. Average autocorrelation spectra of sample cat83, $T$ $=10 \mathrm{~K}$ and $\hbar \omega_{\text {exc. }}=1.73 \mathrm{eV}$, for the spectral intervals defined in Fig. 8 based on $N=12800$ individual spectra. Note that we observe an autocorrelation maximum around $\Delta E=3 \mathrm{meV}$ for the lowenergy interval, while we find a small but significant maximum around $\Delta E=1.5 \mathrm{meV}$ for the high-energy interval. This detail (see dashed rectangle) is magnified in the inset.

regions. As this impression is not really objective, however, we discuss the spatial autocorrelations. As for the spectral autocorrelations, the spatial average of the intensity at a given photon energy is subtracted. We have averaged the two-dimensional autocorrelation of the PL maps like those in Fig. 10(a) over the full $2 \pi$ angle around the center, i.e., $(\Delta x=0, \Delta y=0)$. Two examples for the low and high regions are shown in Fig. 10(b). Their half width at half maximum is depicted for small spectral windows of $2.5 \mathrm{meV}$ width from low to high energies at the bottom of Fig. 8. It becomes obvious that the spatial extent of the autocorrelations in-

(a) low

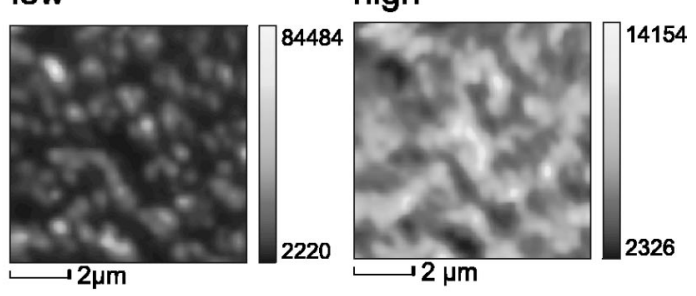

(b)

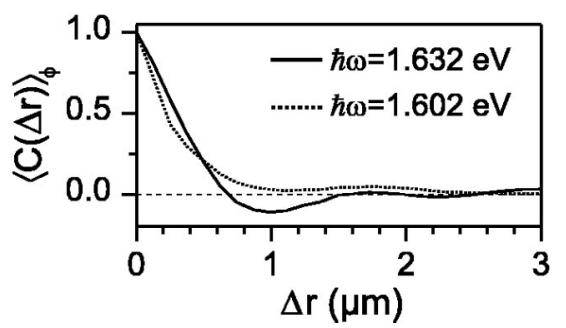

FIG. 10. (a) Photoluminescence maps for sample cat83 for two of the three spectral regions indicated in Fig. 8. Note that the energy interval named low leads to images with well-defined small spots, while the images corresponding to high exhibit more extended bright regions. (b) Spatial autocorrelations (averaged over the full $2 \pi$ angle around the origin) for maps similar to those of (a). The half-width at half maximum $W$ of the spatial autocorrelations throughout the complete spectral interval are shown in Fig. 8. 


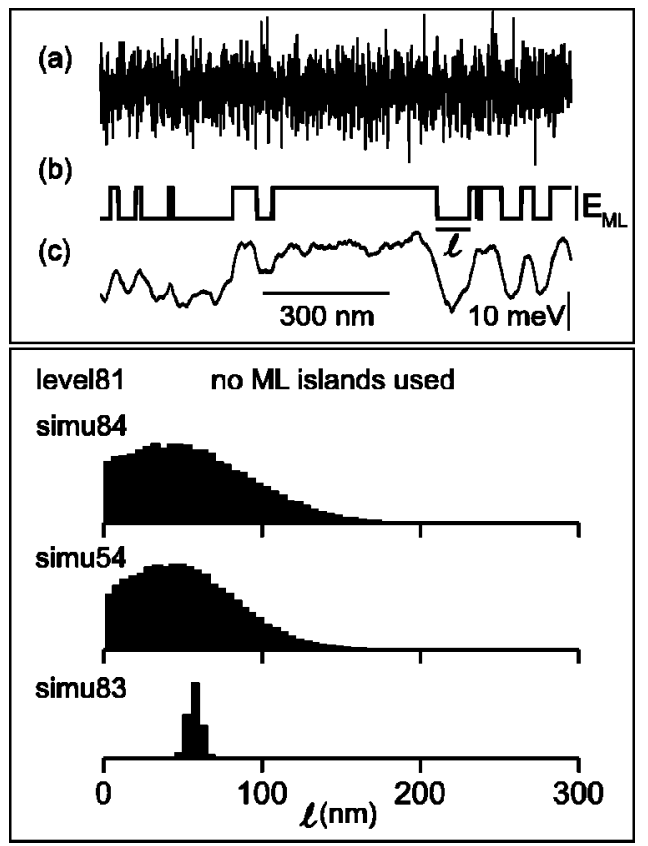

FIG. 11. Calculation: Upper part: Illustration of the disorder potential $V(x)$ resulting from the convolution of the $1 s$ exciton wave function with the sum of a white-noise contribution (a) and monolayer steps (b). This results in the total potential (c), with all potentials plotted on the same energy scale. Lower part: Monolayer island size distribution used for the calculations for the different samples.

creases from low to high energies. In particular, it is much broader for the high region in Fig. 9, where we find the 1.5-meV autocorrelation maximum, which we attribute to level repulsion.

If the $1.5-\mathrm{meV}$ peak does correspond to level repulsion, what is the origin of the $3-\mathrm{meV}$ peak? Sample cat 83 does show obvious signs of monolayer island splitting, as is typical for such thin QW's. Also, note that the sample used in Ref. 1 also showed a shoulder on the low-energy side of the average PL spectrum in Fig. 1 of Ref. 1, corresponding to a

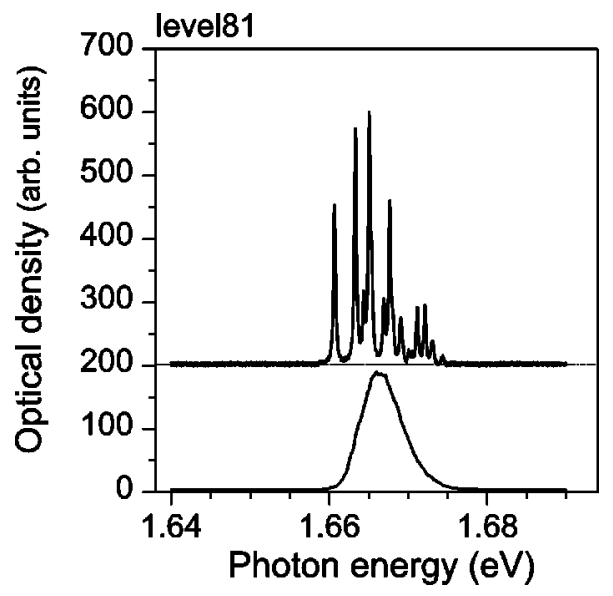

FIG. 12. Calculation (no ML islands): Parameters have been chosen such that both a typical individual (top) and the average (bottom) spectrum match sample cat81 (see Fig. 1), $N=1600$.
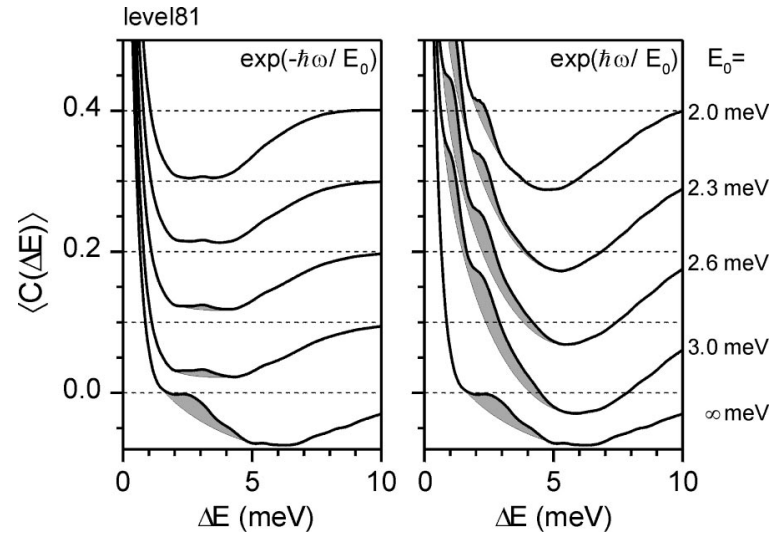

FIG. 13. Calculation (no ML islands): Level repulsion scenario as used in Ref. 1 analyzed with filter functions. The behavior is in striking contrast to our experimental findings on samples cat81 and cat84 (compare with Figs. 2 and 4), $N=1600$.

10-meV monolayer splitting. It is well established ${ }^{5}$ that growth interruption at a GaAs surface leads to extended ML islands on the AlAs-on-GaAs interface. The lateral extent of these islands was determined to be around $40 \mathrm{~nm}$ from PL excitation spectroscopy in Ref. 6. This leads to a lateral quantization energy of about $3 \mathrm{meV}$. If the size of the monolayer islands is not too inhomogeneously distributed, one thus expects a peak of around $3 \mathrm{meV}$ in the autocorrelation spectra. By means of numerical calculations (Sec. III) we will verify that this peak remains even if the standard deviation of the size distribution is comparable to the mean value. It is also well known ${ }^{7}$ that epitaxial growth leads to an atomically rough GaAs-on-AlAs interface which gives rise to a potential contribution resembling white noise. Extended states from these regions as well as from the monolayer islands are responsible for the level repulsion.

\section{NUMERICAL CALCULATIONS}

For a complete modeling of the above photoluminescence experiments - in principle_one not only needs to compute

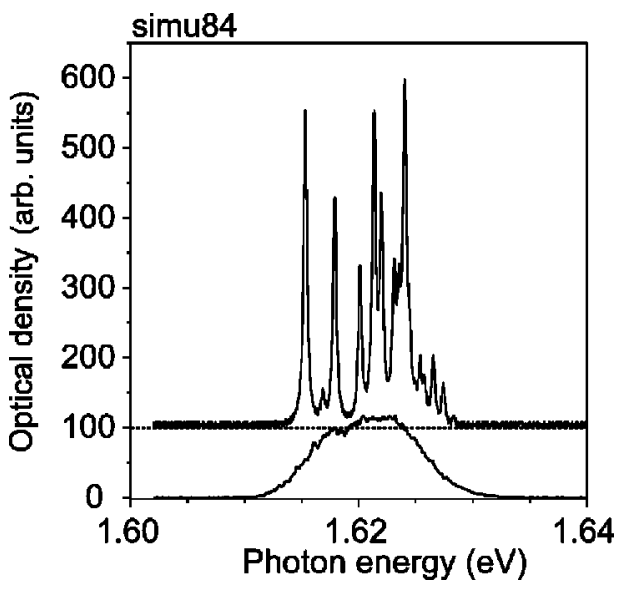

FIG. 14. Calculation (including ML islands): Parameters are chosen such that both a typical individual (top) and the average (bottom) spectrum match sample cat84 (compare with Fig. 3), $\mathrm{N}$ $=1600$. 


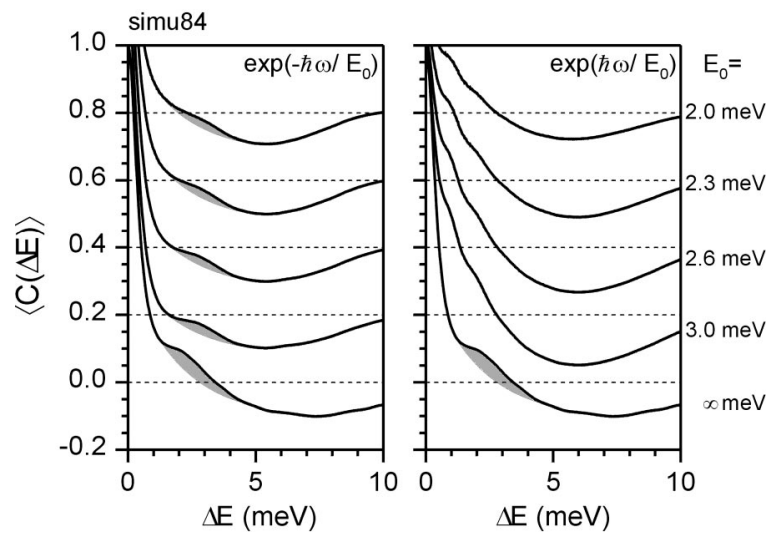

FIG. 15. Calculation (including ML islands): In contrast to Fig. 13, monolayer islands are included. Obviously, this dramatically changes the qualitative behavior. The resulting behavior nicely matches that of the experiment on sample cat 84 (compare with Fig. 4), $N=1600$.

the eigenenergies of excitons in the disorder potential but also treat the kinetics of excitons. If the excitons were thermalized, the photoluminescence spectra would be given by the product of the absorption spectrum and a Boltzmann distribution. Obviously, for a sufficiently high exciton temperature, the absorption and PL would become nearly identical. However, additional experiments ${ }^{9}$ did show several indications that the excitons under these conditions are not thermalized. Adding a streak camera to our setup, we have performed time-resolved experiments which show that the photoluminescence rise times are comparable to the decay times at $T=10 \mathrm{~K}$. Thus excitons cannot fully thermalize under these conditions. In agreement with this observation, numerical calculations ${ }^{10}$ also show that excitons at low temperatures cannot fully thermalize in disordered quantum wells due to the localization in local potential minima.

Complete numerical calculations, including computations of the eigenenergies and the kinetics, have been performed in isolated cases, ${ }^{10,11}$ but can currently not be used in the sense of a fit procedure. Thus such a complete treatment is beyond the scope of this paper. Here-in order to allow for a direct comparison with their work-we strictly follow the lines of Ref. 1, which assumed that the PL spectra are identical to the absorption spectra. While this is a crude assumption indeed, we still expect to obtain at least some general trends and/or features which help to interpret the experiments. In what follows, we compute the eigenenergies $E_{\alpha}$ and the corresponding dipole matrix elements ${ }^{11} M_{\alpha}$, with

TABLE II. Parameters used for the different calculations. For the exciton mass we choose $m=0.3 m_{0}$ (Ref. 11.)

\begin{tabular}{lccc}
\hline \hline data & $E_{\mathrm{ML}}(\mathrm{meV})$ & $\xi(\mathrm{nm})$ & $\sigma(\mathrm{meV})$ \\
\hline level81 & - & 15 & 11.43 \\
simu84 & 5.0 & 17 & 8.89 \\
simu54 & 10.7 & 15 & 7.62 \\
simu83 & 6.5 & 15 & 3.81 \\
\hline \hline
\end{tabular}

$$
M_{\alpha}=\int \Psi_{\alpha}(x) d x,
$$

by numerical solution of the one-dimensional stationary Schrödinger equation

$$
\left(\frac{\hbar^{2}}{2 m} \frac{\partial^{2}}{\partial x^{2}}+V(x)\right) \Psi_{\alpha}(x)=E_{\alpha} \Psi_{\alpha}(x)
$$

for a disorder potential $V(x)$ to be specified now.

Monolayer steps, describing the AlAs-on-GaAs interface, are one contribution to the disorder potential [Fig. 11(b)]. The height $E_{\mathrm{ML}}$ of the ML steps is a parameter (for each sample) which is chosen to match the experimental data. A second parameter associated with the ML steps is the width of the ML islands, $l$. Generally, one expects a distribution of sizes $l$. On the one hand, $l$ cannot be larger than is obvious from the maps (300 $\mathrm{nm}$ resolution limited); on the other hand, it cannot be smaller than the exciton diameter, as the distinct ML peaks in Figs. 5 and 8 would vanish in this case. Apart from the ML islands, the disorder potential contains a second contribution corresponding to the atomically rough GaAs-on-AlAs interface, which is modeled by gaussiandistributed white noise [Fig. 11(a)]. The standard deviation $\sigma$ of this white-noise potential governs the average PL linewidth of both monolayer regions, and is determined this way. The sum of these two potential contributions [(a) and (b) in Fig. 11] has to be convoluted with the exciton wave function to describe the averaging over finer structures due to the inner degrees of freedom of the exciton ${ }^{11}$ [Fig. 11(c)]. Additionally, this convolution also accounts for a certain spatial correlation length $\xi$ in the disorder potential,

$$
V(x) \rightarrow \int_{-\infty}^{+\infty} V\left(x-x^{\prime}\right) \exp \left(-\left|x^{\prime}\right| / \xi\right) d x^{\prime},
$$

and can therefore be seen as another fit parameter. This correlation length $\xi$ was previously used in Ref. 1. The solution of the Schrödinger equation with this disorder potential $V(x)$ delivers the exciton energy levels $E_{\alpha}$ as well as the corresponding dipole matrix elements $M_{\alpha}$ of the optical transitions [see Eq. (1)]. This leads to the optical density $O$ at a photon energy $\hbar \omega$ according to

$$
O(\hbar \omega) \propto \sum_{\alpha} M_{\alpha}^{2} \delta\left(\hbar \omega-E_{\alpha}\right) .
$$

In the numerical calculations, a 300-nm-wide window is chosen to mimic the resolution of the fiber tip in the experiment. ${ }^{12}$ The individual $\delta$-shaped lines in Eq. (4) are convoluted with a Lorentzian with a $300 \mu \mathrm{eV}$ width (corresponding to the average experimental linewidth).

We start our discussion with calculations along the lines of Ref. 1, i.e., with a correlated white-noise potential only (no monolayer islands). The fit parameters are chosen such that the individual and average PL spectra resemble the experimental data of sample cat81 (compare Figs. 12 and 1). At the same time, also the autocorrelation functions (without filter, i.e., for $E_{0}=\infty \mathrm{meV}$ ) agree quite well (compare Figs. 13 and 2). This is the same level of agreement as already 


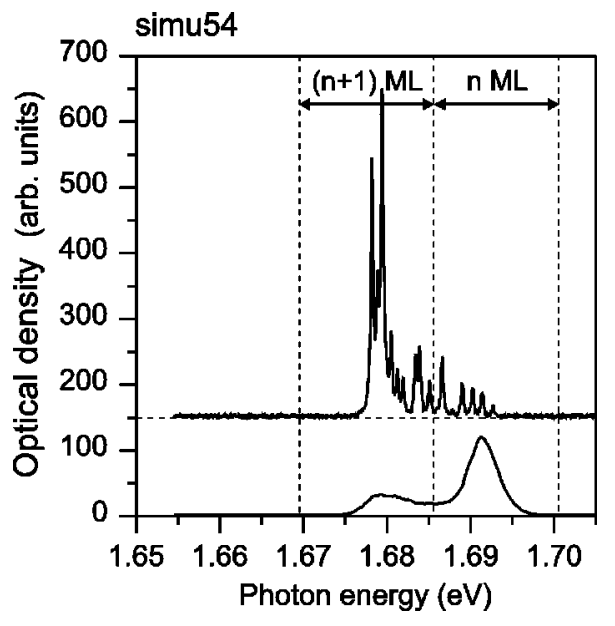

FIG. 16. Calculation (including ML islands): Parameters are chosen such that both a typical individual (top) and the average (bottom) spectrum match sample cat54 (see Fig. 5), $N=1600$.

demonstrated in Ref. 1. However, applying the same filter functions as in the experiment (compare Figs. 13 and 2), we find a striking disagreement between experiment and theory. Consistent with our qualitative discussion on level repulsion in Sec. II, theory shows that the correlation maximum around $3 \mathrm{meV}$ disappears if the low-energy states are emphasized (LHS in Fig. 13), and that it remains if the high-energy states are emphasized (RHS in Fig. 13) - level repulsion stems mostly from the more extended states. This is opposite to the experimental results (see Fig. 2). Thus the theory without monolayer islands is not able to describe the experiment including the filter function test, while theory works fine without the filter functions. This again highlights the crucial importance of the filter function test.

If the correlation maximum in a sample with a small average PL linewidth as cat 81 is related to localized states rather than more extended states, how does and interpret this in sample cat84? As for sample cat81, the calculations without monolayer islands are not able to describe the experimental behavior (not shown). Choosing monolayer islands with a height of $E_{\mathrm{ML}}=5.0 \mathrm{meV}$, the average PL spectrum and the autocorrelation functions resemble the experimental



FIG. 17. Calculation (including ML islands): As in the experiment on sample cat54 (compare with Fig. 6), the correlation maximum in the $(\mathrm{n}+1) \mathrm{ML}$ region is found around $\Delta E=3 \mathrm{meV}$ and $N=1600$.



FIG. 18. Calculation (including ML islands): Parameters are chosen such that both a typical individual (top) and the average (bottom) spectrum match sample cat83 (see Fig. 8), $N=1600$.

findings (compare Figs. 14 and 15 with Figs. 3 and 4): Emphasizing the low-energy states (LHS), the correlation maximum remains, emphasizing the high-energy states (RHS) no distinct structures can be resolved. This is in good qualitative agreement with the experimental data (compare Figs. 4 and 15). From numerous numerical calculations we know that the actual shape of the correlation maximum depends on the precise distribution of the ML island sizes $l$. For sample cat 84 , we have chosen an island size distribution around 40 $\mathrm{nm}$ (see simu84 in Fig. 11), which is in good agreement with experimental findings from photoluminescence excitation spectroscopy on single islands (dots). ${ }^{6}$

Reducing the quantum well width increases the confinement energy and therefore increases the influence of ML fluctuations. Sample cat54 (QW width $3.5 \mathrm{~nm}$ ) shows clearly distinguishable ML regions, which are reproduced in the calculations by increasing the height $E_{\mathrm{ML}}$ of the ML islands (see



FIG. 19. Calculation (including ML islands): As in the experiment on sample cat83 (compare with Fig. 9), the correlation maximum in the low region shifts toward smaller $\Delta E$ due to the wider ML islands as compared to sample cat84. The small and unusual maximum found in the high region (see the inset) nicely reproduces the experiment (see Fig. 9), $N=1600$. This feature is a result of level repulsion. 
Table II). Again the average PL spectrum and the autocorrelation functions resemble the experimental findings (compare Figs. 16 and 17 with Figs. 5 and 6). For the $n+1$ ML region, well-localized states are expected. The autocorrelation function indeed shows a maximum around $\Delta E=3 \mathrm{meV}$ which is more pronounced than for samples cat 81 and cat 84 due to the better spectral separation between the two ML regions. The broad shoulder in the $n$ ML region is also reproduced in the theory (compare $n$ ML regions in Figs. 17 and 6).

Increasing the growth interruption after growing the GaAs quantum well means increasing the monolayer island size and reducing the disorder in the sample, i.e., reducing the amplitude of the white-noise potential contribution. This is exactly what was done in sample cat83 (see Table I). Reducing the white-noise amplitude and increasing the monolayer island size from $40 \mathrm{~nm}$ to $57 \mathrm{~nm}$ in the calculations (see Table II), we are able to qualitatively fit the richly structured average spectrum of sample cat83 (compare Figs. 18 and 8). The various peaks in the average spectrum (Fig. 18) are directly related to the various excited levels corresponding to states localized in one monolayer island potential minimum. The splitting between these states directly corresponds to the (roughly) 3-meV maximum seen in the autocorrelation spectra of the previous samples as well as in this sample (compare low in Fig. 19 with low in Fig. 9). These lines become visible in the average spectrum because the contribution of the white-noise potential is smaller in sample cat83 (see the smaller $\sigma$ in Table II). As a result of the larger average island size $l$ of sample cat 83 (see Table II), the quantization energy in the islands is reduced, which shifts the autocorrelation maximum toward somewhat smaller values of $\Delta E$ as compared to the other samples. Most interestingly, the unusual shape of the autocorrelation functions for the high regions also agrees very well (compare high and insets in Figs. 9 and 19). The wave functions corresponding to these high-energy (PL) lines are not localized in the potential minima of individual monolayer islands. Inspection of the corresponding wave functions (not depicted) shows that they are more extended than the ones corresponding to localization in monolayer islands - in nice agreement with the findings of the experiment [see Fig. 10(b)]. Thus the autocorrelation maximum of the high region not only has a different energetic position than the low region, but also has a different physical origin: It reflects level repulsion inasmuch as one reaches this idealized case in reality. In the strict sense, level repulsion only occurs in a white-noise potential — which we have not found in any of the semiconductor quantum-well samples investigated.

\section{CONCLUSION}

In conclusion we have shown that the recently reported $3-\mathrm{meV}$ peak in the autocorrelation spectrum of nanophotoluminescence spectra of a single GaAs quantum well is not consistent with level repulsion if one takes the data to the test by applying filter functions. These filter functions show that the 3-meV peak is due to low-energy states which we attribute to the lateral quantization of excitons in the monolayer islands. The $1.5-\mathrm{meV}$ peak we observe on the highenergy side is attributed to level repulsion. As expected, the corresponding photoluminescence images show more extended states indeed. In general, one has to be aware that the autocorrelation spectra are not uniquely related to one disorder potential. However, as we have exemplified in this paper, the combination of autocorrelation spectra and various possibilities of filter functions is a powerful tool to determine the underlying disorder potential. In theory, we have used disorder potentials containing both a white-noise contribution and monolayer islands. Interestingly, by slight variations of the parameters, one can generate a rich variety of behaviors, and thus account for many of the surprising features seen in the experiments on different samples.

\section{ACKNOWLEDGMENTS}

This research was supported through the DFG by the Leibniz award (M.W.), the SFB 195, the CFN, the GK 284, and also by the BMBF. The Tucson group acknowledges support from NSF EPDT, JSOP (AFOSR and ARO), and NSA/ARO and the Humboldt Research Program (H.M.G.). We thank Th. Schimmel for stimulating discussions, H. Kalt for the cooperation on the time-resolved experiments, and E. Runge for substantial help in the early phase of the numerical calculations.
*Email address: georg.freymann@physik.uni-karlsruhe.de

${ }^{1}$ F. Intonti, V. Emiliani, C. Lienau, Th. Elsaesser, V. Savona, E. Runge, R. Zimmermann, R. Nötzel, and K. H. Ploog, Phys. Rev. Lett. 87, 076801 (2001).

${ }^{2}$ G. von Freymann, E. Kurtz, C. Klingshirn, and M. Wegener, Appl. Phys. Lett. 77, 394 (2000).

${ }^{3}$ A. Ourmazd, D. W. Taylor, J. Cunningham, and C. W. Tu, Phys. Rev. Lett. 62, 933 (1989).

${ }^{4}$ D. Gammon, B. V. Shanabrook, and D. S. Katzer, Phys. Rev. Lett. 67, 1547 (1991).

${ }^{5}$ R. F. Kopf, E. F. Schubert, T. D. Harris, R. S. Becker, and G. H. Gilmer, J. Appl. Phys. 74, 6139 (1993).

${ }^{6}$ D. Gammon, E. S. Snow, B. V. Shanabrook, D. S. Katzer, and D. Park, Phys. Rev. Lett. 76, 3005 (1996).
${ }^{7}$ D. Bimberg, F. Heinrichsdorff, R. K. Bauer, D. Gerthsen, D. Stenkamp, D. E. Mars, and J. N. Miller, J. Vac. Sci. Technol. B 10, 1793 (1992).

${ }^{8}$ The excessive noise of the detection system leads to a large peak in the autocorrelation functions around $\Delta E=0$. Thus, in the excitation-power-dependent experiments, we have avoided a changing influence of read-out noise (the main source of noise of the CCD camera) by arranging the CCD exposure time such that the product of excitation power and exposure time is constant. If photoluminescence is linear, this leads to a constant number of counts on the camera, hence, to a constant statistics. The total number of spectra of one data set, $N$, is limited in this set of experiments to $N=400$ due to excessive exposure times at the lowest excitation powers. 
${ }^{9}$ U. Neuberth, L. Walter, G. von Freymann, B. Dal Don, H. Kalt, M. Wegener, G. Khitrova, and H. M. Gibbs, Appl. Phys. Lett. 80, 3340 (2002).

${ }^{10}$ S. D. Baranovskii, R. Eichmann, and P. Thomas, Phys. Rev. B 58, 13081 (1998).
${ }^{11}$ E. Runge and R. Zimmermann, Ann. Phys. (N.Y.) 8, 229 (1999); Phys. Status Solidi B 206, 167 (1998).

${ }^{12}$ To avoid artifacts from the boundaries of the 300-nm-wide window, we employ the usual periodic boundary conditions. 